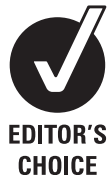

CHOICE

\title{
To RCT or not to RCT: deciding when 'more evidence is needed' for public health policy and practice
}

\author{
Mark Petticrew, ${ }^{1}$ Zaid Chalabi, ${ }^{1}$ David R Jones ${ }^{2}$
}

- An additional figure is published online only. To view this file please visit the journal online (http://jech.bmi.com/ content/66/5.toc)

${ }^{1}$ Public and Environmental Health Research Unit, London School of Hygiene and Tropical Medicine, London, UK ${ }^{2}$ Department of Health Sciences, University of Leicester, Leicester, UK

\section{Correspondence to} Mark Petticrew, London School of Hygiene and Tropical Medicine, 15-17 Tavistock Place, London WC1H 9SH, UK mark.petticrew@Ishtm.ac.uk

Accepted 7 April 2011 Published Online First 7 June 2011

\begin{abstract}
Background Amid the calls for 'more public health evidence', we also need simple understandable methods of determining when more research really is needed. This paper describes a simple decision aid to help policymakers, researchers and other decision makers assess the potential 'information value' of a new public health randomised controlled trial.

Methods The authors developed a flow chart to help make explicit (1) the user's information needs, (2) the intended use of the new information that the study will produce, (3) the added value of the evidence to be derived from the new study and (4) the levels of precision, bias and generalisability required by the user. Results The flow chart is briefly illustrated, first in generic form and then in a worked example, showing how it may be used in deciding whether a new study should be commissioned to evaluate the health impact of allowing motorcycles to use bus lanes in London.

Conclusions In this paper, the authors have presented a flow chart for enacting an informal 'Value-ofInformation'-like approach to deciding when a new public health evaluation is needed. The authors do not suggest that the flow chart approach is technically the equivalent of Value-of-Information methods. Nonetheless, it represents a valuable perspective and process to adopt, and this structured approach will be more revealing than an unstructured thought experiment as the basis for decisions about a new study. To aid in its development as an effective tool, we invite users from a variety of perspectives and contexts to review it, to use it in practice and to send us their comments.
\end{abstract}

\section{INTRODUCTION}

There have been many recent calls for more and better public health $(\mathrm{PH})$ evidence. However, the decision to fund or conduct any new research is itself an intervention, and like any intervention, it needs careful consideration of its costs and benefits. To this end, an informal hierarchy of methods for considering the value of a new study-whether in $\mathrm{PH}$ or other fields - is currently employed, running from unstructured thought experiments, through deterministic and stochastic simulation of a new study, to formal Value-of-Information (VOI) methods. Systematic reviews have also been advocated for identifying needs for further research; but after collecting and summarising existing evidence, the process of identifying the need for more evidence then usually remains informal. There is need for more formal but easily understood approaches to assessing the need for new $\mathrm{PH}$ research, and this paper proposes one such approach, based on a flow chart, which may help consider the value of new $\mathrm{PH}$ trials.

\section{DEMAND VERSUS NEED FOR BETTER PH EVIDENCE}

Many reports in recent years have called for more and better $\mathrm{PH}$ evidence. This evidence can take many forms, and although policymakers and practitioners require many types of qualitative and quantitative evidence to inform decision making, there has been a particular focus on the role and suitability of randomised controlled trials (RCTs) in this context. ${ }^{1-5}$ Demands for rapid development in our knowledge of 'what works' are often seen as synonymous with a need for more RCTs, as opposed to other non-experimental study designs. However, in healthcare, there is often a considerable difference between 'demand' and 'need', and the same applies to research. Demands for new research should be considered carefully, and the costs and benefits carefully assessed. The activities of commissioning, funding and conducting research all have costs and benefits, not least opportunity costs because the budget to fund $\mathrm{PH}$ research is not unlimited, and there are constraints in the capacity of the research community. Researchers claiming that more 'research is needed', and commissioners of new research need simple and effective ways of assessing the potential gains from new research.

This assessment can be particularly difficult for $\mathrm{PH}$ research, where the outcomes of trials may legitimately include costs and benefits in sectors other than health (such as education, transport and other sectors), as well as improvements in knowledge and reductions in uncertainty among decision makers. Although formal methods for assessment of the balance of costs and benefits of a new study (such as VOI approaches) are important, ${ }^{6}$ they may also be difficult in many current $\mathrm{PH}$ contexts. For example, in $\mathrm{PH}$, decision modelling to predict the effects of interventions may be more complex than in clinical therapeutic contexts, covering a wider scope (from incidence to death), and may require significantly more data to populate the models. $\mathrm{PH}$ also takes in sectors other than the health sector, and so, the impacts of $\mathrm{PH}$ programmes may need to be modelled in terms of housing, employment, crime and other improvements, as well as their health effects. The available data may not be framed in ways that can be easily used; as noted, there are fewer trials, and there is greater emphasis on the use of non-experimental, observational and qualitative evidence. There is also the problem of incommensurability, that is, the difficulty in valuing/weighting different outcomes across 
different sectors in the same units, and the need to consider equity issues.

As an alternative, or in some cases as a preliminary to formal VOI approaches, we present below an approach based on a flow chart to help PH decision makers at all levels consider whether a new study is needed, and if so, what sort of study is most worthwhile. We have focused here on the need for more RCTs because that is where the $\mathrm{PH}$ 'evidence gap' is often suggested to lie; there have been many papers and reports about the lack of trials, but few or none about the lack of qualitative research, or observational research. First, however, we consider what influences the perceived usefulness of research.

\section{WOULD BETTER EVIDENCE BE USED?}

One key audience for any new PH RCTs and other studies is policymakers. We therefore need to consider whether new research, if available, would actually be used by this group. Here, the evidence is mixed: robust research methodology is not always an important consideration in whether policymakers use research findings or not. A systematic review examining health policymakers' use of evidence found that the main facilitators of research evidence in policymaking are personal contact, timeliness, clear recommendations, good quality research, confirmatory research and community pressure for research. ${ }^{7}$ By contrast, research that included effectiveness data (such as RCTs) was mentioned in only 3 studies out of 24 .

The perceived value of RCTs also varies between sectors. One UK policy advisor, experienced in using RCTs, and an advocate of their wider use for social policy, has noted: 'By and large, methodology is a weak influence, in the sense that policy makers don't really tend to weigh up research evidence in terms of the strength of the source, it's much more the signal that they're interested in...The influence of research findings is primarily a function of those findings, rather than the methods'.

This observation is consistent with a growing literature showing that in $\mathrm{PH}$, as in other fields, the use of evidence is influenced by many factors, few of which are methodological. This leaves researchers and research commissioners with a challenge. There is ample evidence that the $\mathrm{PH}$ evidence base is weak, but new research should not simply contribute to what has been termed avoidable waste in the production of research. ${ }^{9}$

\section{WHEN DO WE HAVE 'ENOUGH' EVIDENCE?}

In some cases, it will be clear that we already have 'enough' evidence, even if we have no trials; where the signal is large relative to the background noise, observational data alone may suffice. ${ }^{10}$ In practical terms, a high signal to noise ratio can be expected when the effect size is large and when there is rapidity of change in subjective and objective outcome measures. An RCT to detect such a clear effect is probably not required, while in other situations, an RCT may be feasible but may be neither necessary or appropriate. ${ }^{11}$

We also need to consider the strength, as well as the type of evidence that is required. For example, the NICE methods guidance on $\mathrm{PH}$ evidence describes the most appropriate evidence to inform different types of $\mathrm{PH}$ decision $^{12}$ (table 1).

\section{FORMAL APPROACHES TO PRIORITISING THE COLLECTION OF FUTURE PH EVIDENCE}

Although the literature on how policymakers use evidence goes back more than a century, ${ }^{13}$ it is largely concerned with the use of existing evidence, rather than with how future research priorities may be established. A hierarchy of methods for considering the value of a new study from it already exists. ${ }^{14} 15$ This runs from an unstructured thought experiment, through deterministic and stochastic simulation of new study, to formal VOI methods. ${ }^{6}$ Systematic reviews have also often been advocated as an effective means of identifying needs for further research. ${ }^{14}$ However, although they embody formal methods for summarising existing evidence, the process of identifying the need for more evidence thereafter remains largely undefined.

More formal methods have received less attention in practice. One of the most promising approaches, VOI methods, allows formalised and quantified identification of the contribution of particular pieces of information in decision models. The potential costs of obtaining sample information in a further study and the potential benefits of information so gained may be considered explicitly in terms of improved precision.

Nonetheless, problems arise in applying current VOI approaches in $\mathrm{PH}$. The impacts of $\mathrm{PH}$ programmes often need to be modelled in terms of effects in housing, employment, crime and other sectors, as well as their health effects. Problems of incommensurability result: valuing/weighting different outcomes across different sectors in the same units is required. Equity issues also need to be considered, ${ }^{16}$ though in principle exploration of differences between subgroups is possible. ${ }^{17}$ In $\mathrm{PH}$, decision modelling to predict the effects of interventions may be more complex than in clinical therapeutic contexts, requiring significantly more data to populate the models. Finally, few researchers and policymakers are likely to have the necessary capacity, skills and resources to conduct some VOI analyses. ${ }^{18}$

Table 1 Appropriateness of different types of evidence for answering public health questions (reproduced with permission, National Institute for Health and Clinical Excellence (NICE), 2009)

\begin{tabular}{|c|c|c|c|c|c|c|}
\hline & \multicolumn{6}{|l|}{ Type of evidence } \\
\hline & $\begin{array}{l}\text { Systematic review } \\
\text { of effectiveness (and } \\
\text { cost-effectiveness) }\end{array}$ & $\begin{array}{l}\text { Experimental } \\
\text { study }\end{array}$ & Observational study & Qualitative study & $\begin{array}{l}\text { Practice/ } \\
\text { case report }\end{array}$ & $\begin{array}{l}\text { Economic/cost- } \\
\text { effectiveness study }\end{array}$ \\
\hline $\begin{array}{l}\text { Extent of public health } \\
\text { problem/issue }\end{array}$ & & & $\begin{array}{l}\text { NICE epidemiological } \\
\text { review }\end{array}$ & & & \multirow{4}{*}{$\begin{array}{l}\text { NICE cost-effectiveness } \\
\text { review }\end{array}$} \\
\hline $\begin{array}{l}\text { Factors/determinants/ } \\
\text { associations }\end{array}$ & NICE review of reviews & & NICE correlates review & & & \\
\hline $\begin{array}{l}\text { Views and experiences } \\
\text { of practitioners }\end{array}$ & & & NICE correlates review & NICE qualitative review & \multirow[t]{2}{*}{$\begin{array}{l}\text { NICE mapping } \\
\text { report }\end{array}$} & \\
\hline $\begin{array}{l}\text { Views and experiences of } \\
\text { target population }\end{array}$ & & & NICE correlates review & NICE qualitative review & & \\
\hline
\end{tabular}


Figure 1 Generic flow chart. RCT, randomised controlled trial. (Continued)

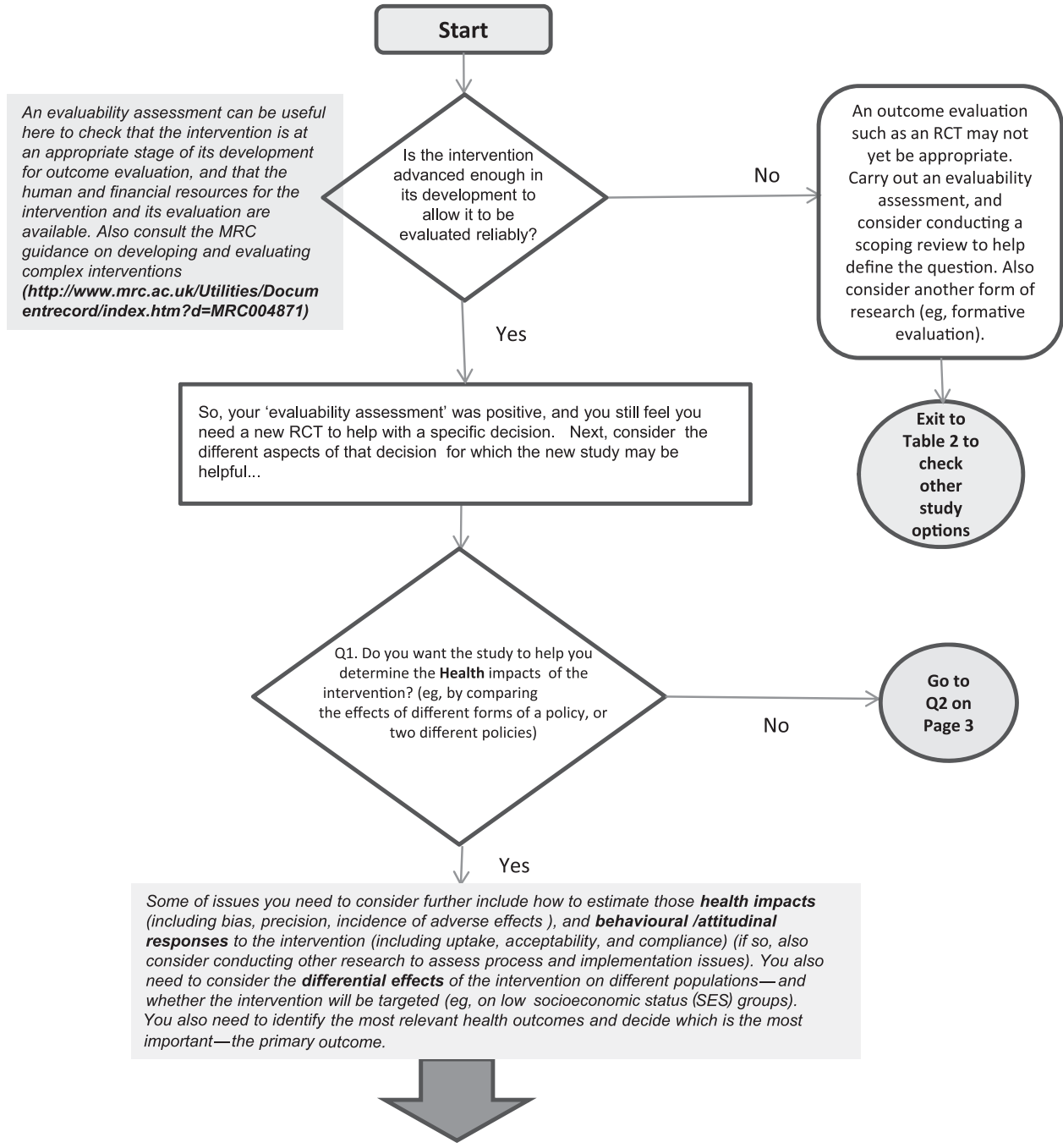

\section{TOWARDS AN INFORMAL APPROACH TO ASSESSING THE VALUE OF NEW INFORMATION}

Where a full VOI analysis is not feasible, or not regarded as worthwhile, a structured approach to deciding whether a new study is needed in $\mathrm{PH}$ contexts can be helpful. Here, a flow chart may yield some of the benefits of the VOI approach more easily. The example we have developed is targeted principally at $\mathrm{PH}$ decision makers in policy and implementation contexts but will also be useful to commissioners and funders of $\mathrm{PH}$ research, and researchers themselves, to inform decisions about future research activities.

Use of the flow chart (figure 1) requires making explicit (1) the user's information needs and (2) the intended use of the new information that the study will produce. The added value of the evidence to be derived from the new study and the levels of precision, bias and generalisability required are then considered.

Use of the flow chart is briefly illustrated, first in generic form (figure 1) and then in a worked example, showing how it may be used in deciding whether a new study should be commissioned to evaluate the health impact of allowing motorcycles to use bus lanes in London. (A further two examples will be made available in the web-only materials relating to (1) introducing new environmental standards in residential homes for elderly people and (2) to exercise prescribing by general practitioners.)

\section{The generic framework}

The framework (figure 1) begins by asking the user to consider whether the intervention is really ready to be evaluated; since evaluation at too early a stage in their development has been suggested as a reason why $\mathrm{PH}$ interventions fail to demonstrate effectiveness. ${ }^{19}$ A range of approaches have been developed to help evaluators assess the 'evaluation readiness' of new interventions. ${ }^{20}$ After this, the framework encourages the user to think through what they may learn from a new trial, about first the health impacts (see Q1 in figure 1) and then the non-health impacts of the new intervention ( $\mathrm{Q} 2$ in figure 1). The latter could include impacts on employment or educational opportunities. For example, an intervention that promotes physical activity in people unable to work may improve individual health, but it may also increase an individual's ability to return to work-which may in turn have health impacts.

The user is also asked to consider whether, and how, ambiguous and/or unambiguous research findings from the study would affect future decision making. If their decision would not be affected by an ambiguous research finding, then the value of future information from a new RCT may be low. However, evidence from some other study design may be valuable, and so users are routed to table 2 (adapted from Muir Gray ${ }^{21}$ ) to consider what other research options are available to them. If, having progressed through the flow chart, the user still feels that a new RCT will be of value, then other considerations are 
Figure 1 Continued

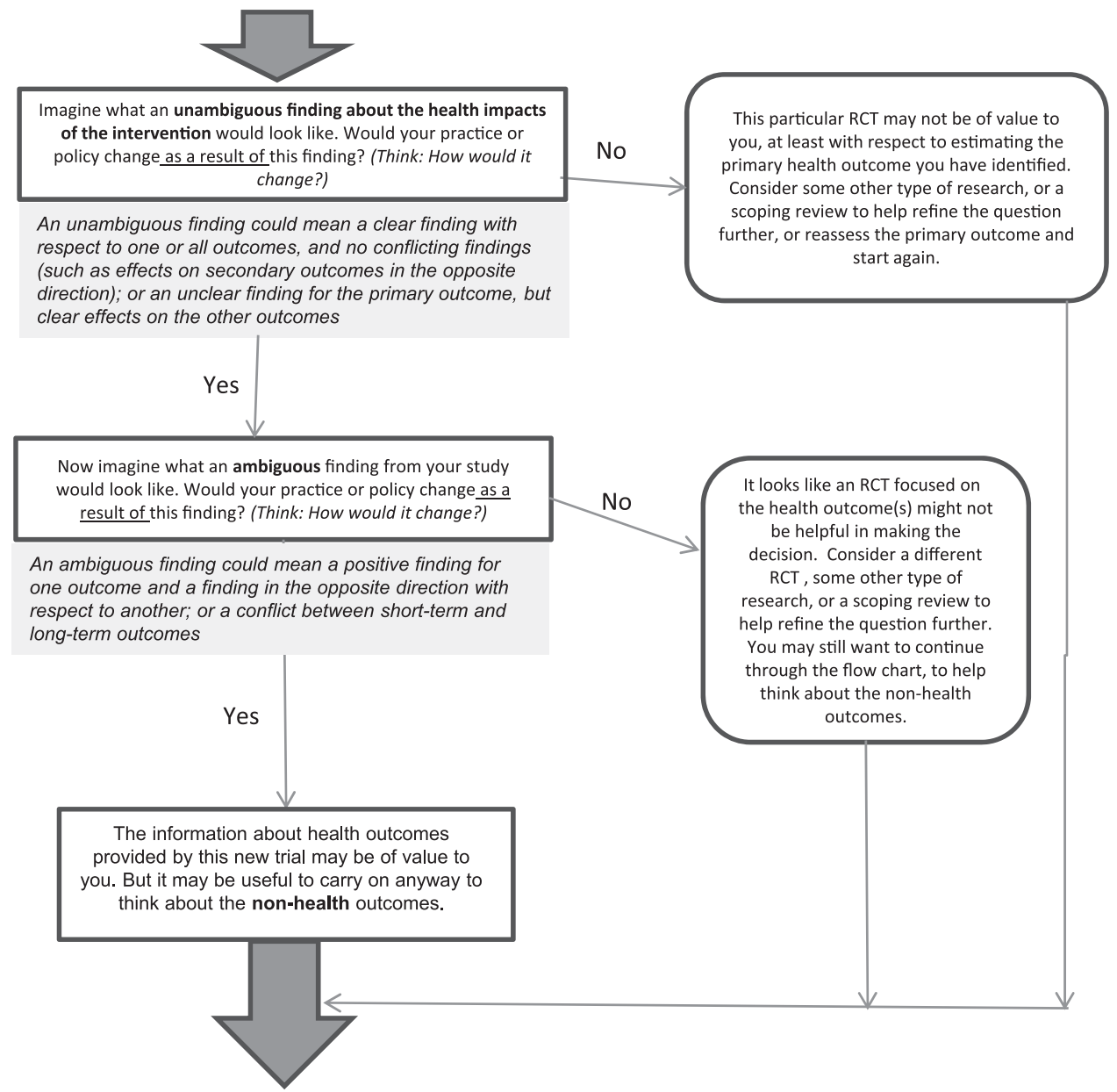

presented in the (blue) commentary boxes, such as the need to consider healthy equity and adverse effects.

\section{Worked example: health impacts of allowing motorcycles to use bus lanes}

Online figure 1 shows an application of the flow chart to a hypothetical transport policy question: 'Should an RCT be commissioned to evaluate the impact of allowing motorcyclists to use bus lanes in London?' To start with, the user is asked whether this traffic management intervention is at an appropriate stage for a trial evaluation. The evaluability issues could include the practicality of doing a trial in different parts of London and the resources required. The user is directed through the flow chart if, and only if, they deem that the intervention is appropriate for a trial. Otherwise they are invited to consider other study options such as conducting interviews with stakeholders or carrying out reviews to check whether relevant evidence is available, say, from trials in other European cities (such as Rome or Athens where motorcycles are in common use).

If users regard the intervention as appropriate for evaluation in a trial, they are next asked whether they consider that the trial is required in order to determine the health impacts. If so, they are then invited to consider how to estimate those impacts, say in terms of the rate and severity of traffic-related injuries, and whether to disaggregate these impacts across different road users (cyclists, pedestrians, motorcyclists). If not concerned with the future health impacts, users are directed towards consideration of non-health impacts, such as the costs of implementation, the impact on traffic congestion and the acceptability of the intervention to road users.

Whether interested in the health impacts or the non-health impacts, users are asked in each case to imagine what an unambiguous finding and an ambiguous finding would look like. An unambiguous finding about health impacts might include the reduction of traffic-related injuries for all road users. Nonhealth impacts might include finding the intervention is costeffective, reduces traffic congestion and is well received by all road users. On the other hand, an ambiguous finding about health impacts could arise where the intervention reduces traffic-related injuries for one type of road users (eg, motorcyclists) but increases it for another (eg, cyclists); ambiguous nonhealth impacts could arise if the intervention reduces traffic congestion but is costly and unacceptable to London bus drivers.

The flow chart will guide users in deciding whether the trial would be of value (for evaluating health and/or non-health impacts). If not, they are asked to consider other study options or to re-assess the outcomes, perhaps through assigning relative weights to each of the outcomes following elicitation of the views of stakeholders, say, before going through the flow chart again.

\section{DISCUSSION}

In this paper, we have presented a flow chart for enacting an informal VOI-like approach to deciding when a new $\mathrm{PH}$ trial is needed. Formulation as a decision problem emphasises the importance of the decision determining what information is 
Figure 1 Continued

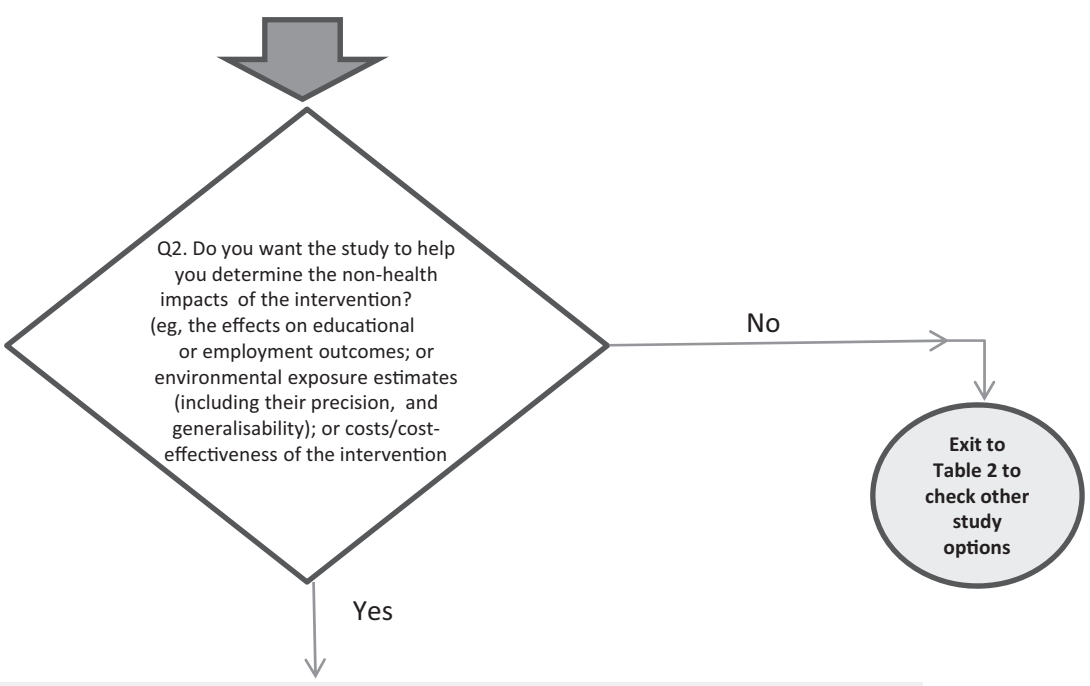

\begin{abstract}
Then some of issues you need to consider further include how to estimate those impacts (including bias, precision, incidence of adverse effects ). You may also need to consider the differential effects of the

intervention on different populations - for example will it impact on health inequalities?The PROGRESS framework will be useful to help consider this issue when designing a study (http://equity.cochrane.org/). Also consider unintended effects (such as spillover and ripple effects, and adverse effects)
\end{abstract}

Imagine what an unambiguous finding from your study would look like. Consider how your practice or policy would change as a result of this finding

Now imagine what an ambiguous finding from your study would look like. Would your practice or policy change as a result of this finding? (Think: How would it change?)

One's practice or policy may or may not change even in the light of new positive or negative (or ambiguous or unambiguous) findings. If study results are ambiguous with respect to the primary outcome then the decision may rest on the relative weight given to the other health and non-health outcomes, as well ass issues such as the cost, and acceptability of the intervention. Top help with such decisions it may be helpful to be explicit about the relative weights given to different outcomes. However it may also be the case that if your decision is not affected by the findings of this new RCT that you don't need the study after all. If this is the case you might also want to go to Table 2 to check other study options before proceeding.

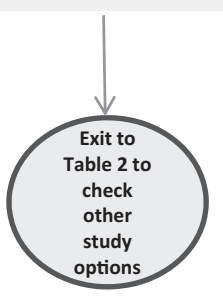

needed to make it, rather than allowing the set of information available to determine what decisions are made. ${ }^{22}$

In most cases, we expect the flow chart will need to be used iteratively; a first pass through the chart will reveal

Table 2 Appropriateness of different research designs to different types of question (adapted from Muir Gray ${ }^{21}$ )

\begin{tabular}{|c|c|c|c|c|c|c|}
\hline & Qualitative research & Surveys & Case control & Cohort & RCT & Systematic review \\
\hline Effectiveness of an intervention & & & & & $\sqrt{ } \sqrt{ }$ & $\sqrt{ } \sqrt{ }$ \\
\hline Effectiveness of health service delivery & $\sqrt{ }$ & $\sqrt{ }$ & $\sqrt{ }$ & $\sqrt{ }$ & $\sqrt{ } \sqrt{ }$ & $\sqrt{ } \sqrt{ }$ \\
\hline Safety & $\sqrt{ }$ & $\sqrt{ }$ & & & $\sqrt{ } \sqrt{ }$ & $\sqrt{ } \sqrt{ }$ \\
\hline Acceptability & $\sqrt{ }$ & $\sqrt{ }$ & & & $\sqrt{ } \sqrt{ }$ & $\sqrt{ } \sqrt{ }$ \\
\hline Cost-effectiveness & & & & & $\sqrt{ } \sqrt{ }$ & $\sqrt{ } \sqrt{ }$ \\
\hline Appropriateness & $\sqrt{ }$ & $\sqrt{ }$ & & & & $\sqrt{ } \sqrt{ }$ \\
\hline Quality & $\sqrt{ }$ & $\sqrt{ }$ & $\sqrt{ }$ & $\sqrt{ }$ & & $\sqrt{ } \sqrt{ }$ \\
\hline
\end{tabular}

issues-perhaps health outcomes or costs, say-not initially considered and hence needing to be worked through in a second pass, and so on. In most cases, it will be helpful to draw a decision tree summarising the outcomes in the example. As we

$\mathrm{RCT}$, randomised controlled trial. 
have already indicated, it is not claimed that the informal approach here will reach the same finishing point as a VOI analysis or indeed a multi-criteria decision analysis approach ${ }^{23}$; however, in some cases, it may be apparent that one decision option dominates the others since it provides better benefits at lower cost. ${ }^{24}$ As always in contexts such as these, sensitivity analyses to check assumptions made are essential; it may be that here informal critical threshold analyses can be performed to indicate how extreme cost or benefit estimates would need to be to throw the optimality of a particular decision into doubt. ${ }^{24}$ Another result of using the approach here may be that a more formal VOI analysis will be seen to be worthwhile.

We are not, of course, suggesting that our flow chart approach is technically the equivalent of VOI methods. Nonetheless, we do regard it as representing a valuable perspective and process to adopt, and more likely to be feasible than VOI per se, at least in $\mathrm{PH}$. In almost all cases, the structured approach will be more revealing than an unstructured thought experiment as the basis for decisions about a new study.

In seeking to keep the flow chart as simple as possible, we will inevitably have omitted or oversimplified some issues, and in focusing on evidence of effectiveness, we have not discussed the importance of context, and the likelihood that policymakers will require not just evidence of whether interventions work, but also whether, and how they work in different contexts. ${ }^{12}$ To aid in its development as an effective tool, we invite users of it from a variety of perspectives and contexts to review it, to use it in practice and to send us their comments, either directly by email

\section{What is already known on this subject}

There is ongoing debate about the need for more robust public health research, particularly more RCTs.

- However, researchers and commissioners of new research also need simple and effective ways of assessing the potential gains from any new research.

- Current approaches, such as VOI approaches to estimating the payback from new studies, can be technically challenging and are not transparent to users.

- An additional approach is needed.

\section{What this study adds}

- We propose a novel flow chart-based approach to assessing when new research is needed, and what type of study may be most useful.

- It incorporates consideration of health and non-health outcomes and may be of particular use in decisions about the value of public health RCTs.

- In almost all cases, the structured approach we propose will be more revealing than an unstructured 'thought experiment' as the basis for decisions about a new study. or on a blog set up for this purpose: http://publichealthevidence. blogspot.com/.

Acknowledgements This paper is based in part on work done when DRJ was on sabbatical leave from the University of Leicester in 2009.

Competing interests MP and DRJ are members of the National Institute of Health Research Public Health Research Funding Board; DRJ was previously a member of the NICE Public Health Interventions Advisory Board.

Contributors The authors wrote this paper as equal contributors following discussions about the use of formal VOl approaches in public health and having worked together on previous grant applications on the topic. This led to the development of the approach (and the supporting examples, which Zaid Chalabi led on the development of) in a series of meetings in 2009. DRJ and MP had also previously been involved in a grant application on VOI in public health in 2006.

Provenance and peer review Not commissioned; externally peer reviewed.

\section{REFERENCES}

1. Wanless D. Securing Good Health for the Whole Population: Final Report. London: HM Treasury, 2004. http://www.hmtreasury.gov.uk/consultations and legislation/ wanless/consult wanless04 final.cfm.

2. Public Health Sciences: Challenges and opportunities. A Report of the Public Health Sciences Working Group Convened by the Wellcome Trust. London: Wellcome Trust, 2004.

3. Mackenzie M, O'Donnell C, Halliday $E$, et al. Evaluating complex interventions: one size does not fit all. BMJ 2010:340:401-3.

4. Oxman A, Bjørndal A, Becerra-Posada F, et al. A framework for mandatory impact evaluation to ensure well informed public policy decisions. Lancet 2010;375:427-31.

5. Anon. Evaluation: the top priority for global health. Lancet 2010;375:526.

6. Claxton K, Sculpher M. Using value of information analysis to prioritise health research: some lessons from recent UK experience. Pharmacoeconomics 2006;24:1055-68.

7. Innvaer S, Vist G, Trommald M, et al. Health policy-makers' perceptions of their use of evidence: a systematic review. J Health Serv Res Policy 2002;7:239-44.

8. Roberts H, Petticrew M, Macintyre S, et al. Randomised Controlled Trials of Social Interventions: Report of a Pilot Study of Barriers and Facilitators in An International Context. Glasgow, Scotland: Medical Research Council Social and Public Health Sciences Unit (MRC SPHSU), 2009. Occasional Paper No. 19.

9. Chalmers I, Glasziou P. Avoidable waste in the production and reporting of research evidence. BMJ 2009;374:86-9.

10. Glasziou $\mathbf{P}$, Rawlins $\mathrm{M}$. When are randomised trials unnecessary? Picking signal from noise. BMJ 2007;334:349-51.

11. Black N. Why we need observational studies to evaluate the effectiveness of health care. BMJ 1996;312:1215-18.

12. Methods for the development of NICE Public health guidance. London: NICE, 2009.

13. Nutley S, Webb L. Evidence and the Policy process. What Works? Evidence Based Policy and Practice in Public Services. Bristol: Policy Press, 2000.

14. Sutton A, Cooper N, Jones D. Evidence synthesis as the key to more coherent and efficient research. BMC Med Res Methodol 2009;9:29.

15. Sutton A, Cooper N, Jones D, et al. Evidence-based sample size calculations based upon updated meta-analysis. Stat Med 2007;26:2479-500.

16. Potvin L. Yes! More research is needed; but not just any research. Int J Public Health 2009;54:127-8.

17. Epstein D, Chalabi Z, Claxton K, et al. Efficiency, equity, budgetary policies: informing decision using mathematical programming. Med Decis Making 2007;27:128-37.

18. Ades A, Lu G, Claxton K. Expected value of sample information calculations in medical decision modelling. Med Decis Making 2004;24:207-27.

19. Hawe P, Shiell A, Riley T. Complex interventions: how "out of control" can a randomised controlled trial be? BMJ 2004:328:1561-3.

20. Youtie J, Bozeman B, Shapira P. Using an evaluability assessment to select methods for evaluating state technology development programs: the case of the Georgia Research Alliance. Eval Program Plann 1999;22:55-64

21. Muir Gray JA. Evidence-Based Healthcare. London: Churchill Livingstone, 1997.

22. Dowie J. Health impact: its estimation, assessment and analysis. In: Orme J, Powell Taylor P, Harrison T, Grey M, eds. Public Health for the 21st Century: New Perspectives on Policy, Participation and Practice. Buckingham: Open University Press, 2003:296-309.

23. Baltussen R, Niessen L. Priority setting of health interventions: the need for multicriteria decision analysis. Cost Eff Resour Alloc 2006;4:14. http://www.resourceallocation.com/content/4/1/14.

24. Haddix A, Teutsch S, Corso P. Prevention Effectiveness. A Guide to Decision Analysis and Economic Evaluation. 2nd edn. Oxford: Oxford University Press, 2003. 